\title{
Article
}

\section{Some applications of second-order differential subordination for a class of analytic function defined by the lambda operator}

\author{
B. Venkateswarlu ${ }^{1, *}$, P. Thirupathi Reddy ${ }^{2}$, S. Sridevi ${ }^{1}$ and Sujatha ${ }^{1}$ \\ 1 Department of Mathematics, GSS, GITAM University, Doddaballapur- 562 163, Bengaluru Rural, Karnataka, India. \\ 2 Department of Mathematics, Kakatiya Univeristy, Warangal- 506 009, Telangana, India. \\ * Correspondence: bvlmaths@gmail.com
}

Received: 12 October 2020; Accepted: 17 December 2020; Published: 27 December 2020.

Abstract: In this paper, we introduce a new class of analytic functions by using the lambda operator and obtain some subordination results.

Keywords: Analytic, convex, subordination, symmetric.

MSC: 30C45.

\section{Introduction}

$\mathbf{L}$ et $\mathbb{C}$ be complex plane and let $\mathbb{U}=\{z: z \in \mathbb{C}$ and $|z|<1\}=\mathbb{U} \backslash\{0\}$ be an open unit disc in $\mathbb{C}$. Also let $H(\mathbb{U})$ be a class of analytic functions in $\mathbb{U}$. For $n \in \mathbb{N}=\{1,2,3, \cdots$,$\} and a \in \mathbb{C}$, let $H[a, n]$ be a subclass of $H(\mathbb{U})$ formed by the functions of the form

$$
f(z)=z+a_{n} z^{n}+a_{n+1} z^{n+1}+\cdots
$$

with $H_{0} \equiv H[0,1]$ and $H \equiv H[1,1]$. Suppose that $A_{n}$ is a class of all analytic functions of the form

$$
f(z)=z+\sum_{k=n+1}^{\infty} a_{n} z^{n}
$$

in the open unit disk $\mathbb{U}$ with $A_{1}=A$. A function $f \in H(\mathbb{U})$ is univalent if it is a one-to-one function in $\mathbb{U}$. By $S$, we denote a subclass of $A$ formed by functions univalent in $\mathbb{U}$. If a function $f \in A$ maps $\mathbb{U}$ onto a convex domain and $f$ is univalent, then $f$ is called a convex function. By

$$
K=\left\{f \in A: \Re\left\{1+\frac{z f^{\prime \prime}(z)}{f^{\prime}(z)}\right\}>0, z \in \mathbb{U}\right\},
$$

we denote a class of all convex functions defined in $\mathbb{U}$ and normalized by $f(0)=0$ and $f^{\prime}(0)=1$.

Let $f$ and $F$ be elements of $H(\mathbb{U})$. A function $f$ is said to be subordinate to $F$, if there exists a Schwartz function $w$ analytic in $\mathbb{U}$ with $w(0)=0$ and $|w(z)|<1, z \in \mathbb{U}$, such that $f(z)=F(w(z))$. In this case, we write $f(z) \prec F(z)$ or $f \prec F$. Furthermore, if the function $F$ is univalent in $\mathbb{U}$, then we get the following equivalence [1,2]:

$$
f(z) \prec F(z) \Leftrightarrow f(0)=F(0) \text { and } f(\mathbb{U}) \prec F(\mathbb{U}) .
$$

The method of differential subordinations (also known as the method of admissible functions) was first introduced by Miller and Mocanu in 1978 [3], and the development of the theory was originated in 1981 [4]. All details can be found in the book by Miller and Mocanu [2]. In recent years, numerous authors studied the properties of differential subordinations (see [5-8], etc.).

Let $\Psi: \mathbb{C}^{3} \times \mathbb{U} \rightarrow \mathbb{C}$ and let $h$ be univalent in $\mathbb{U}$. If $p$ is analytic in $\mathbb{U}$ and satisfies the second-order differential subordination:

$$
\Psi\left(p(z), z p^{\prime}(z), z p^{\prime \prime}(z) ; z\right) \prec h(z),
$$


then $p$ is called the solution of differential subordination. The univalent function $q$ is called a dominant of the solution of the differential subordination or, simply, a dominant if $p \prec q$ for all $p$ satisfying (2). The dominant $q_{1}$ satisfying $q_{1} \prec q$ for all dominants $q$ of (2) is called the best dominant of (2).

Let us recall lambda function [9] defined by:

$$
\lambda(z, s)=\sum_{k=2}^{\infty} \frac{z^{k}}{(2 k+1)^{k}}
$$

where $z \in \mathbb{U}, s \in \mathbb{C}$, when $|z|<1, \Re(s)>1$, when $|z|=1$ and let $\lambda^{(-1)}(z, s)$ be defined such that

$$
\lambda(z, s) * \lambda^{(-1)}(z, s)=\frac{1}{(1-z)^{\mu+1}}, \mu>-1
$$

We now define $\left(z \lambda^{(-1)}(z, s)\right)$ as:

$$
(z \lambda(z, s)) *\left(z \lambda^{(-1)}(z, s)\right)=\frac{z}{(1-z)^{\mu+1}}=z+\sum_{k=2}^{\infty} \frac{(\mu+1)_{k-1}}{(k-1) !} z^{k}, \mu>-1
$$

and obtain the linear operator $\mathcal{I}_{\mu}^{s} f(z)=\left(z \lambda^{(-1)}(z, s)\right) * f(z)$, where $f \in A, z \in \mathbb{U}$ and $\left(z \lambda^{(-1)}(z, s)\right)=$ $z+\sum_{k=2}^{\infty} \frac{(\mu+1)_{k-1}(2 k-1)^{s}}{(k-1) !} z^{k}$. A simple computation gives us

$$
\mathcal{I}_{\mu}^{s} f(z)=z+\sum_{k=2}^{\infty} L(k, \mu, s) a_{k} z^{k}
$$

where

$$
L(k, \mu, s)=\frac{(\mu+1)_{k-1}(2 k-1)^{s}}{(k-1) !}
$$

where $(\mu)_{k}$ is the Pochhammer symbol defined in terms of the Gamma function by:

$$
(\mu)_{k}=\frac{\Gamma(\mu+k)}{\Gamma(\mu)}= \begin{cases}1, & \text { if } k=0 \\ \mu(\mu+1) \cdots(\mu+k-1), & \text { if } k \in \mathbb{N}\end{cases}
$$

Definition 1. Let $\mathfrak{L}_{\mu, s}(\varrho)$ be a class of function $f \in A$ satisfying the inequality

$$
\Re\left(\mathcal{I}_{\mu}^{s} f(z)\right) \geq \varrho,
$$

where $z \in \mathbb{U}, 0 \leq \varrho<1$ and $\mathcal{I}_{\mu}^{s} f(z)$ is the Lambda operator.

Lemma 1. let $h$ be a convex function with $h(0)=a$ and let $\gamma \in \mathbb{C}^{*}:=\mathbb{C} \backslash\{0\}$ be a complex number with $\Re\{\} \geq 0$. If $p \in H[a, n]$ and

$$
p(z)+\frac{1}{\gamma} z p^{\prime}(z) \prec h(z),
$$

then $p(z) \prec q(z) \prec h(z)$, where $q(z)=\frac{\gamma}{n z^{\frac{\gamma}{n}}} \int_{0}^{z} t^{\frac{\gamma}{n-1}} h(t) d t, \quad z \in \mathbb{U}$. The function $q$ is convex and is the best dominant for subordination (5).

Lemma 2. [10] Let $\Re\{\}>0, n \in \mathbb{N}$ and $w=\frac{n^{2}+|\mu|^{2}-\left|n^{2}-\mu^{2}\right|}{4 n \Re\{\}}$. Also, let $h$ be an analytic function in $\mathbb{U}$ with $h(0)=1$. Suppose that $\Re\left\{1+\frac{z h^{\prime \prime}(z)}{h^{\prime}(z)}\right\}>-w$. If $p(z)=1+p_{n} z^{n}+p_{n+1} z^{n+1}+\cdots$ is analytic in $\mathbb{U}$ and

$$
p(z)+\frac{1}{\mu} z p^{\prime}(z) \prec h(z),
$$


then $p(z) \prec q(z)$, where $q$ is a solution of the differential equation $q(z)+\frac{n}{\mu} z q^{\prime}(z)=h(z), q(0)=1$, given by $q(z)=\frac{\mu}{n z^{\frac{\mu}{n}}} \int_{0}^{z} t^{\frac{\mu}{n-1}} h(t) d t, z \in \mathbb{U}$. Moreover, $q$ is the best dominant for the differential subordination (6).

Lemma 3. [11] Let $r$ be a convex function in $\mathbb{U}$ and let $h(z)=r(z)+n \varrho z r^{\prime}(z), \quad z \in \mathbb{U}$, where $\varrho>0$ and $n \in \mathbb{N}$. If $p(z)=r(0)+p_{n} z^{n}+p_{n+1} z^{n+1}+\cdots, z \in \mathbb{U}$, is holomorphic in $\mathbb{U}$ and $p(z)+\varrho z p^{\prime}(z) \prec h(z), z \in \mathbb{U}$, then $p(z) \prec r(z)$ and this result is sharp.

In the present paper, we use the subordination results from [10] to prove our main results.

\section{Main results}

Theorem 1. The set $\mathfrak{L}_{\mu, s}(\varrho)$ is convex.

Proof. Let $f_{j}(z)=z+\sum_{k=2}^{\infty} a_{k, j} z^{k}, z \in \mathbb{U}, j=1, \cdots, m$ be in the class $\mathfrak{L}_{\mu, s}(\varrho)$. Then, by Definition 1 , we get

$$
\Re\left\{\left(\mathcal{I}_{\mu}^{s} f(z)\right)^{\prime}\right\}=\Re\left\{1+\sum_{k=2}^{\infty} L(k, \mu, s) a_{k, j} k z^{k-1}\right\}>\varrho .
$$

For any positive numbers $\varsigma_{1}, \varsigma_{2}, \varsigma_{3}, \cdots, \varsigma_{m}$ such that $\sum_{j=1}^{m} \varsigma_{j}=1$, it is necessary to show that the function $h(z)=\sum_{j=1}^{m} \varsigma_{j} f_{j}(z)$ is an element of $\mathfrak{L}_{\mu, s}(\varrho)$, i.e.,

$$
\Re\left\{\left(\mathcal{I}_{\mu}^{s} h(z)\right)^{\prime}\right\}>\varrho .
$$

Thus, we have

$$
\mathcal{I}_{\mu}^{s} h(z)=z+\sum_{k=2}^{\infty} L(k, \mu, s)\left\{\sum_{j=1}^{m} \varsigma_{j} a_{k, j}\right\} z^{k} .
$$

If we differentiate (9) with respect to $z$, then we obtain

$$
\left(\mathcal{I}_{\mu}^{s} h(z)\right)^{\prime}=1+\sum_{k=2}^{\infty} k L(k, \mu, s)\left\{\sum_{j=1}^{m} \varsigma_{j} a_{k, j}\right\} z^{k-1} .
$$

Thus by using (8), we have

$$
\Re\left\{\left(\mathcal{I}_{\mu}^{s} h(z)\right)^{\prime}\right\}=1+\sum_{j=1}^{m} \varsigma_{j} \Re\left\{\sum_{k=2}^{\infty} k L(k, \mu, s) a_{k, j} z^{k-1}\right\}>1+\sum_{j=1}^{m} \varsigma_{j}(\varrho-1)=\varrho .
$$

Hence, inequality (7) is true and we arrive at the desired result.

Theorem 2. Let $q$ be convex function in $\mathbb{U}$ with $q(0)=1$ and $h(z)=q(z)+\frac{1}{\gamma+1} z q^{\prime}(z), z \in \mathbb{U}$, where $\gamma$ is a complex number with $\Re\{\gamma\}>-1$. If $f \in \mathfrak{L}_{\mu, s}(\varrho)$ and $\aleph=\mathrm{Y}_{\gamma} f$, where

$$
\aleph(z)=\mathrm{Y}_{\gamma} f(z)=\frac{\gamma+1}{z^{\gamma}} \int_{0}^{z} t^{\gamma-1} f(t) d t
$$

then

$$
\left(\mathcal{I}_{\mu}^{s} f(z)\right)^{\prime} \prec h(z)
$$

implies that $\left(\mathcal{I}_{\mu}^{s} \aleph(z)\right)^{\prime} \prec q(z)$ and this result is sharp. 
Proof. In view of equality (10), we can write

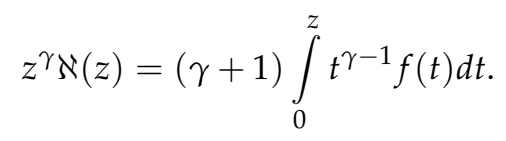

Differentiating (12) with respect to $z$, we obtain $(\gamma) \aleph(z)+z \aleph^{\prime}(z)=(\gamma+1) f(z)$. Further, by applying the operator $\mathcal{I}_{\mu}^{s}$ to the last equation, we get

$$
(\gamma) \mathcal{I}_{\mu}^{s} \aleph(z)+z\left(\mathcal{I}_{\mu}^{s} \aleph(z)\right)^{\prime}=(\gamma+1) \mathcal{I}_{\mu}^{s} f(z) .
$$

If we differentiate (13) with respect to $z$, then we find

$$
\left(\mathcal{I}_{\mu}^{s} \aleph(z)\right)^{\prime}+\frac{1}{\gamma+1} z\left(\mathcal{I}_{\mu}^{s} f(z)\right)^{\prime \prime}=\left(\mathcal{I}_{\mu}^{s} f(z)\right)^{\prime} .
$$

By using the differential subordination given by (11) in equality (14), we obtain

$$
\left(\mathcal{I}_{\mu}^{s} \aleph(z)\right)^{\prime}+\frac{1}{\gamma+1} z\left(\mathcal{I}_{\mu}^{s} f(z)\right)^{\prime \prime} \prec h(z) .
$$

We define

$$
p(z)=\left(\mathcal{I}_{\mu}^{s} \aleph(z)\right)^{\prime} .
$$

Hence, as a result of simple computations, we get

$$
p(z)=\left\{z+\sum_{k=2}^{\infty} L(k, \mu, s) \frac{\gamma+1}{\gamma+k} a_{k} z^{k}\right\}^{\prime}=1+p_{1} z+p_{2} z^{2}+\cdots, p \in H[1,1] .
$$

By using (16) in subordination (15), we obtain

$$
p(z)+\frac{1}{\gamma+1} z p^{\prime}(z) \prec h(z)=q(z)+\frac{1}{\gamma+1} z q^{\prime}(z), \quad z \in \mathbb{U} .
$$

If we use Lemma 2, then we write $p(z) \prec q(z)$. Thus, we obtained the desired result and $q$ is the best dominant.

Example 1. If we choose $\gamma=i+1$ and $q(z)=\frac{1+z}{1-z}$, in Theorem 2, then we get $h(z)=\frac{(i+2)-((i+2) z+2) z}{(i+2)(1-z)^{2}}$. If $f \in \mathfrak{L}_{\mu, s}(\varrho)$ and $\aleph$ is given as $\aleph(z)=Y_{i} f(z)=\frac{i+2}{z^{i+1}} \int_{0}^{z} t^{i} f(t) d t$, then, by virtue of Theorem 2 , we find $\left(\mathcal{I}_{\mu}^{s} f(z)\right)^{\prime} \prec$ $h(z)=\frac{(i+2)-((i+2) z+2) z}{(i+2)(1-z)^{2}}$, implies $\left(\mathcal{I}_{\mu}^{s} f(z)\right)^{\prime} \prec \frac{1+z}{1-z}$.

Theorem 3. Let $\Re\{\gamma\}>-1$ and $w=\frac{1+|\gamma+1|^{2}-\left|\gamma^{2}+2 \gamma\right|}{4 \Re\{\gamma+1\}}$. Suppose that $h$ is an analytic function in $\mathbb{U}$ with $h(0)=1$ and that $\Re\left\{1+\frac{z h^{\prime \prime}(z)}{h^{\prime}(z)}\right\}>-w$. If $f \in \mathfrak{L}_{\mu, s}(\varrho)$ and $\aleph=Y_{\mu}^{s} f$, where $\aleph$ is defined by (10), then

$$
\left(\mathcal{I}_{\mu}^{s} f(z)\right)^{\prime} \prec h(z)
$$

implies that $\left(\mathcal{I}_{\mu}^{s} \aleph(z)\right)^{\prime} \prec q(z)$, where $q$ is the solution of the differential equation $h(z)=q(z)+\frac{1}{\gamma+1} z q^{\prime}(z), q(0)=1$, given by $q(z)=\frac{\gamma+1}{z^{\gamma+1}} \int_{0}^{z} t^{\gamma} f(t) d t$. Moreover, $q$ is the best dominant for subordination (17).

Proof. If we choose $n=1$ and $\mu=\gamma+1$ in Lemma 1, then the proof is obtained by means of the proof of Theorem 3.

Theorem 4. Let

$$
h(z)=\frac{1+(2 \varrho-1) z}{1+z}, 0 \leq \varrho<1
$$


be convex in $\mathbb{U}$ with $h(0)=1$. If $f \in A$ and verifies the differential subordination $\left(\mathcal{I}_{\mu}^{s} f(z)\right)^{\prime} \prec h(z)$, then $\left(\mathcal{I}_{\mu}^{s} \aleph(z)\right)^{\prime} \prec$ $q(z)=(2 \varrho-1)+\frac{2(1-\varrho)(\gamma+1) \tau(\gamma)}{z^{\gamma+1}}$, where $\tau$ is given by the formula

$$
\tau(\gamma)=\int_{0}^{z} \frac{t^{\gamma}}{t+1} d t
$$

and $\aleph$ is given by equation (10). The function $q$ is convex and is the best dominant.

Proof. If $h(z)=\frac{1+(2 \varrho-1) z}{1+z}, 0 \leq \varrho<1$, then $h$ is convex and, in view of Theorem 3, we can write $\left(\mathcal{I}_{\mu}^{s} \aleph(z)\right)^{\prime} \prec$ $q(z)$. Now, by using Lemma1, we get

$$
q(z)=\frac{\gamma+1}{z^{\gamma+1}} \int_{0}^{z} t^{\gamma} h(t) d t=\frac{\gamma+1}{z^{\gamma+1}} \int_{0}^{z} t^{\gamma}\left\{\frac{1+(2 \varrho-1) t}{1+t}\right\} d t=(2 \varrho-1)+\frac{2(1-\varrho)(\gamma+1)}{z^{\gamma+1}} \tau(\gamma),
$$

where $\tau$ is given by (19). Hence, we obtain

$$
\left(\mathcal{I}_{\mu}^{s} \aleph(z)\right)^{\prime} \prec q(z)=(2 \varrho-1)+\frac{2(1-\varrho)(\gamma+1) \tau(\gamma)}{z^{\gamma+1}} .
$$

The function $q$ is convex. Moreover, it is the best dominant. Hence the theorem is proved.

Theorem 5. If $0 \leq \varrho<1,0 \leq \mu<1, \delta \geq 0, \Re\{\gamma\}>-1$, and $\aleph=\mathrm{Y}_{\gamma} f$ is defined by (10), then $\mathrm{Y}_{\gamma}\left(\mathfrak{L}_{\mu, s}(\varrho)\right) \subset$ $\mathfrak{L}_{\mu, s}(\rho)$, where

$$
\rho=\min _{|z|=1} \Re\{q(z)\}=\rho(\gamma, \varrho)=(2 \varrho-1)+2(1-\varrho)(\gamma+1) \tau(\gamma)
$$

and $\tau$ is given by (19).

Proof. Assume that $h$ is given by equation (18), $f \in \mathfrak{L}_{\mu, s}(\varrho)$, and $\aleph=\mathrm{Y}_{\gamma} f$ is defined by (10). Then $h$ is convex and, by Theorem 3, we deduce

$$
\left(\mathcal{I}_{\mu}^{S} \aleph(z)\right)^{\prime} \prec q(z)=(2 \varrho-1)+\frac{2(1-\varrho)(\gamma+1) \tau(\gamma)}{z^{\gamma+1}},
$$

where $\tau$ is given by (19). Since $q$ is convex, $q(\mathbb{U})$ is symmetric about the real axis, and $\Re\{\gamma\}>-1$, we find

$$
\Re\left\{\left(\mathcal{I}_{\mu}^{s} \aleph(z)\right)^{\prime}\right\} \geq \min _{|z|=1} \Re\{q(z)\}=\Re\{q(1)\}=\rho(\gamma, \varrho)=(2 \varrho-1)+2(1-\varrho)(\gamma+1)(1-\varrho) \tau(\gamma) .
$$

It follows from inequality (21) that $\mathrm{Y}_{\gamma}\left(\mathfrak{L}_{\mu, s}(\varrho)\right) \subset \mathfrak{L}_{\mu, s}(\rho)$, where $\rho$ is given by (20). Hence the theorem is proved.

Theorem 6. Let $q$ be a convex function with $q(0)=1$ and $h$ be a function such that $h(z)=q(z)+z q^{\prime}(z), z \in \mathbb{U}$. If $f \in A$, then the subordination

$$
\left(\mathcal{I}_{\mu}^{s} f(z)\right)^{\prime} \prec h(z)
$$

implies that $\frac{\mathcal{I}_{\mu}^{s} f(z)}{z} \prec q(z)$, and the result is sharp.

Proof. Let

$$
p(z)=\frac{\mathcal{I}_{\mu}^{s} f(z)}{z} .
$$

Differentiating (23), we find $\left(\mathcal{I}_{\mu}^{s} f(z)\right)^{\prime}=p(z)+z p^{\prime}(z)$. We now compute $p(z)$. This gives

$$
p(z)=\frac{\mathcal{I}_{\mu}^{s} f(z)}{z}=\frac{z+\sum_{k=2}^{\infty} L(k, \mu, s) a_{k} z^{k}}{z}=1+p_{1} z+p_{2} z^{2}+\cdots, \quad p \in H[1,1] .
$$


By using (24) in subordination (22), we find $p(z)+z p^{\prime}(z) \prec h(z)=q(z)+z q^{\prime}(z)$. Hence, by applying Lemma 3, we conclude that $p(z) \prec q(z)$ i.e., $\frac{\mathcal{I}_{\mu}^{s} f(z)}{z} \prec q(z)$. This result is sharp and $q$ is the best dominant. Hence the theorem is proved.

Example 2. If we take $\mu=0$ and $s=1$ in equality (4) and $q(z)=\frac{1}{1-z}$ in Theorem 5 , then $h(z)=\frac{1}{(1-z)^{2}}$ and

$$
I_{0}^{1} f(z)=z+\sum_{k=2}^{\infty} \frac{(2 k-1)}{(k-1) !} a_{k} z^{k}
$$

Differentiating (25) with respect to $z$, we get

$$
\left(I_{0}^{1} f(z)\right)^{\prime}=1+\sum_{k=2}^{\infty} \frac{(2 k-1)}{(k-1) !} a_{k} z^{k-1}=1+p_{1} z+p_{2} z^{2}+\cdots, p \in H[1,1] .
$$

By using Theorem 5, we find $\left(I_{0}^{1} f(z)\right)^{\prime} \prec h(z)=\frac{1}{(1-z)^{2}}$. This yields $\frac{I_{0}^{1} f(z)}{z} \prec q(z)=\frac{1}{1-z}$.

Theorem 7. Let $h(z)=\frac{1+(2 \varrho-1) z}{1+z}, z \in \mathbb{U}$ be convex in $\mathbb{U}$ with $h(0)=1$ and $0 \leq \varrho<1$. If $f \in$ A satisfies the differential subordination

$$
\left(\mathcal{I}_{\mu}^{s} f(z)\right)^{\prime} \prec h(z),
$$

then $\frac{\mathcal{I}_{\mu}^{s} f(z)}{z} \prec q(z)=(2 \varrho-1)+\frac{2(1-\varrho) \ln (1+z)}{z}$. The function $q$ is convex and, in addition, it is the best dominant.

Proof. Let

$$
p(z)=\frac{\mathcal{I}_{\mu}^{s} f(z)}{z}=1+p_{1} z+p_{2} z^{2}+\cdots, p \in H[1,1] .
$$

Differentiating (27), we find

$$
\left(\mathcal{I}_{\mu}^{s} f(z)\right)^{\prime}=p(z)+z p^{\prime}(z)
$$

In view of (28), the differential subordination (26) becomes $\left(\mathcal{I}_{\mu}^{s} f(z)\right)^{\prime} \prec h(z)=\frac{1+(2 \varrho-1) z}{1+z}$, and by using Lemma 1 , we deduce $p(z) \prec q(z)=\frac{1}{z} \int h(t) d t=(2 \varrho-1)+\frac{2(1-\varrho) \ln (1+z)}{z}$. Now, by virtue of relation (27) we obtained the desired result.

Corollary 1. If $f \in \mathfrak{L}_{\mu, s}(\varrho)$, then $\Re\left(\frac{\mathcal{I}_{\mu}^{s} f(z)}{z}\right)>(2 \varrho-1)+2(1-\varrho) \ln (2)$.

Proof. If $f \in \mathfrak{L}_{\mu, s}(\varrho)$, then it follows from Definition 1 that $\Re\left\{\left(\mathcal{I}_{\mu}^{s} f(z)\right)^{\prime}\right\}>\varrho, z \in \mathbb{U}$, which is equivalent to $\left(\mathcal{I}_{\mu}^{s} f(z)\right)^{\prime} \prec h(z)=\frac{1+(2 \varrho-1) z}{1+z}$. Now, by using Theorem 7 , we obtain

$$
\frac{\mathcal{I}_{\mu}^{s} f(z)}{z} \prec q(z)=(2 \varrho-1)+\frac{2(1-\varrho) \ln (1+z)}{z} .
$$

Since $q$ is convex and $q(\mathbb{U})$ is symmetric about the real axis, we conclude that

$$
\Re\left(\frac{\mathcal{I}_{\mu}^{s} f(z)}{z}\right)>\Re(q(1))=(2 \varrho-1)+2(1-\varrho) \ln (2) .
$$

Theorem 8. Let $q$ be a convex function such that $q(0)=1$ and $h$ be the function given by the formula $h(z)=q(z)+$ $z q^{\prime}(z), z \in \mathbb{U}$. If $f \in A$ and verifies the differential subordination

$$
\left\{\frac{z \mathcal{I}_{\mu}^{s} f(z)}{\mathcal{I}_{\mu}^{s} \aleph(z)}\right\}^{\prime} \prec h(z), \quad z \in \mathbb{U},
$$


then $\frac{\mathcal{I}_{\mu}^{s} f(z)}{\mathcal{I}_{\mu}^{s} \aleph(z)} \prec q(z), \quad z \in \mathbb{U}$, and this result is sharp.

Proof. For function $f \in A$, given by Equation (1), we get

$$
\mathcal{I}_{\mu}^{s} \aleph(z)=z+\sum_{k=2}^{\infty} L(k, \mu, s) \frac{\gamma+1}{k+\gamma} a_{k} b_{k} z^{k}, \quad z \in \mathbb{U} .
$$

We now consider the function

$$
p(z)=\frac{\mathcal{I}_{\mu}^{s} f(z)}{\mathcal{I}_{\mu}^{s} \aleph(z)}=\frac{z+\sum_{k=2}^{\infty} L(k, \mu, s) a_{k} b_{k} z^{k}}{z+\sum_{k=2}^{\infty} L(k, \mu, s) \frac{\gamma+1}{k+\gamma} a_{k} b_{k} z^{k}}=\frac{1+\sum_{k=2}^{\infty} L(k, \mu, s) a_{k} b_{k} z^{k-1}}{1+\sum_{k=2}^{\infty} L(k, \mu, s) \frac{\gamma+1}{k+\gamma} a_{k} b_{k} z^{k-1}} .
$$

In this case, we get

$$
(p(z))^{\prime}=\frac{\left(\mathcal{I}_{\mu}^{s} f(z)\right)^{\prime}}{\mathcal{I}_{\mu}^{s} \aleph(z)}-p(z) \frac{\left(\mathcal{I}_{\mu}^{s} \aleph(z)\right)^{\prime}}{\mathcal{I}_{\mu}^{s} \aleph(z)} .
$$

Then

$$
p(z)+z p^{\prime}(z)=\left\{\frac{z \mathcal{I}_{\mu}^{s} f(z)}{\mathcal{I}_{\mu}^{s} \aleph(z)}\right\}^{\prime}, z \in \mathbb{U} .
$$

By using relation (30) in inequality (29), we obtain $p(z)+z p^{\prime}(z) \prec h(z)=q(z)+z q^{\prime}(z)$ and, by virtue of Lemma 3, $p(z) \prec q(z)$, i.e., $\frac{\mathcal{I}_{\mu}^{s} f(z)}{\mathcal{I}_{\mu}^{s} \aleph(z)} \prec q(z)$. Hence the theorem is proved.

Acknowledgments: The authors warmly thank the referees for the careful reading of the paper and their comments.

Author Contributions: All authors contributed equally to the writing of this paper. All authors read and approved the final manuscript.

Conflicts of Interest: "The authors declare no conflict of interest."

\section{References}

[1] Bulboacă, T. (2005). Differential subordinations and superordinations: Recent results. Casa Cărtii de Stiintă.

[2] Miller, S. S., \& Mocanu, P. T. (2000). Differential Subordinations: Theory and Applications. CRC Press.

[3] Miller, S. S., \& Mocanu, P. T. (1978). Second order differential inequalities in the complex plane. Journal of Mathematical Analysis and Applications, 65(2), 289-305.

[4] Miller, S. S., \& Mocanu, P. T. (1981). Differential subordinations and univalent functions. The Michigan Mathematical Journal, 28(2), 157-172.

[5] Akgül, A. (2017). On second-order differential subordinations for a class of analytic functions defined by convolution. Journal of Nonlinear Sciences and Application, 10, 954-963.

[6] Lupas, A. A. (2012). Certain differential subordinations using Salagean and Ruscheweyh operators. Acta Universitatis Apulensis, 29, 125-129.

[7] Bulut, S. (2014). Some applications of second-order differential subordination on a class of analytic functions defined by Komatu integral operator. International Scholarly Research Notices, 2014, Artical ID 606235.

[8] Oros, G. I., \& Oros, G. (2008). On a class of univalent functions defined by a generalized Salagean operator. Complex Variables and Elliptic Equations, 53(9), 869-877.

[9] Spanier, J., \& Oldham, K. B. (1987). An Atlas of Functions. New York: Hemisphere publishing corporation.

[10] Oros, G., \& Oros, G. I. (2003). A class of holomorphic functions II. Libertas Mathematica, 23, 65-68.

[11] Salagean, G. S. (1983). Subclasses of univalent functions. In Complex AnalysisŮFifth Romanian-Finnish Seminar (pp. 362-372). Springer, Berlin, Heidelberg. 